\title{
Nuclear Reaction and Structure Databases of the National Nuclear Data Center
}

\section{B. Pritychenko*}

Brookhaven National Laboratory

Upton, NY 11973, USA

E-mail: pritychenko@bnl.gov

\section{M.W. Herman}

Brookhaven National Laboratory

Upton, NY 11973, USA

E-mail: mwherman@bnl.gov

\section{S.F. Mughabghab}

Brookhaven National Laboratory

Upton, NY 11973, USA

E-mail: mugabgab@bnl.gov

P. Oblozinsky

Brookhaven National Laboratory

Upton, NY 11973, USA

E-mail: oblozinsky@bnl.gov

\section{A.A. Sonzogni \\ Brookhaven National Laboratory \\ Upton, NY 11973, USA \\ E-mail: sonzogni@bnl.gov}

We discuss nuclear data resources of the National Nuclear Data Center (NNDC) of relevance to nuclear astrophysics applications. These resources include databases, tools and powerful web service at www.nndc.bnl.gov. Our objective is to provide an overview of nuclear databases, related products and demonstrate nuclear astrophysics potential of the ENDF/B-VII beta2 library. Discussion on the Maxwellian neutron capture cross sections obtained from the ENDF/B-VII beta2 library is presented.

International Symposium on Nuclear Astrophysics - Nuclei in the Cosmos - IX

CERN, Geneva, Switzerland

25-30 June, 2006

\section{${ }^{*}$ Speaker}




\section{Introduction}

Access to nuclear data is crucial for both basic science and applied nuclear technology communities, including nuclear structure physics, astrophysics, reactors, accelerator design, nonproliferation and safeguards, radiation protection and medicine as well as homeland security applications.

The mission of the National Nuclear Data Center (NNDC) includes collection, evaluation, and dissemination of nuclear physics data for basic nuclear research and applied nuclear technologies [1,2]. The NNDC maintains and contributes to the nuclear reaction (ENDF, CSISRS alias EXFOR), nuclear structure (ENSDF) and bibliography (NSR) databases along with several other databases (NuDat, CapGam). The Center produces several publications such as Atlas of Neutron Resonances [3] and nuclear model codes.

\section{Nuclear Databases}

Recently, nuclear data services were substantially improved to provide better access to reaction data evaluations and compilations [2]. New Web interfaces for the Evaluated Nuclear Data File (ENDF) and the nuclear reaction experimental data (CSISRS/EXFOR) provide a wide range of options for data retrievals and analysis using standard and interpreted text formats as well as graphic tools. New nuclear structure and bibliography Web services (ENSDF, NuDat, NSR) distinguish themselves the simple manner of creating on-line database queries and the extensive use of plots. A front page of the NNDC nuclear data Web services is shown in Figure 1 and a brief description of the nuclear databases is presented below. 


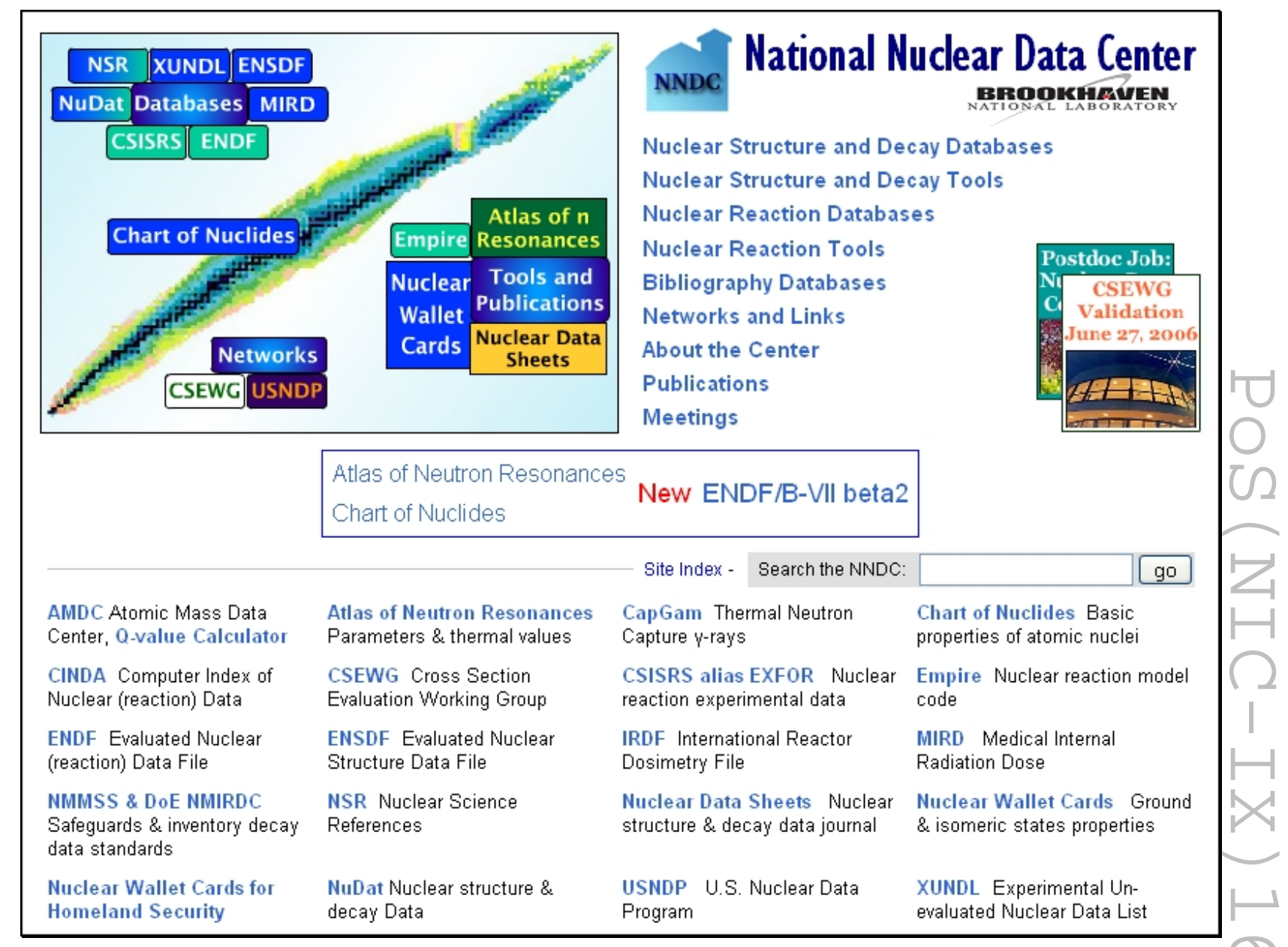

Figure 1. Front page of the NNDC Web Services (www.nndc.bnl.gov). All elements of the page, including graphic images, are hyperlinked.

\subsection{Nuclear Science References}

Nuclear Science References database ( $w w w . n n d c . b n l . g o v / n s r)$ is a starting point for nuclear physics research and application developments. It covers more than 80 journals and offers some 180,000 references, providing an extremely effective way of searching scientific literature on a broad range of nuclear physics topics. In 2004, NSR usability was improved by the addition of 35,000 new digital object identifier (doi) links that allow users to access actual publications directly from the Web.

\subsection{Experimental Nuclear Reaction Data}

Cross Section Information Storage \& Retrieval System (CSISRS), alias EXFOR (EXchange FORmat) data library [1, 2] contains experimental nuclear reaction data for incident neutrons, charged particles, and photons. It includes more than 15500 compiled experiments and covers nearly all of neutron-induced reaction experimental data up to the pion threshold. The library is less complete for charged particle induced reactions (in general A $<12$ ) and photon experiments. The content of the CSISRS database (www.nndc.bnl.gov/exfor) serves as a principal input for ENDF nuclear data evaluations. 


\subsection{Evaluated Nuclear (reaction) Data File}

The ENDF reaction database contains evaluated data from the United States ENDF/BVI.8 library. Presently, the ENDF nuclear reaction database is under new development [4]. The latest version of the library ENDF/B-VII beta2 was released on April 25, 2006 for CSEWG members (www.nndc.bnl.gov/endf2). It contains neutron cross sections for 393 materials (isotopes) relevant to nuclear technology, including capture in the $\mathrm{keV}$ region of interest to astrophysics. Timeline for ENDF/B-VII neutron materials evaluations spans over 30 years with 195 evaluations performed in the last three years. This library also includes 8 nuclear data standards, 20 thermal scattering kernels, 48 proton, 163 photo-nuclear, 3830 decay and 10 charged-particle evaluations. We expect the final version of ENDF/B-VII library and a new Web application to be publicly available in 2006.

Nuclear astrophysics applications of the ENDF nuclear data library include neutron capture cross sections which can be applied for s-process nucleosynthesis [5]. The ENDF/B-VII beta2 library contains the latest neutron evaluated data from $10^{-5} \mathrm{eV}$ to $20 \mathrm{MeV}$. Nuclear astrophysics s-process calculations require Maxwellian average neutron capture cross sections [6]:

$$
\sigma_{\gamma}(E)=\frac{2}{\sqrt{\pi}} \frac{\int_{0}^{\infty} \sigma \gamma(E) E \exp \left(-\frac{E}{k T}\right) d E}{\int_{0}^{\infty} E \exp \left(-\frac{E}{k T}\right) d E},
$$

where $\sigma_{\gamma}(E)$ is the pointwise capture cross section, $E$ is the neutron energy and $k T$ is the thermal energy at temperature $\mathrm{T}$.

Evaluated neutron capture cross sections for the entire ENDF/B-VII beta2 nuclear data library were processed with ENDF utility code INTER [7]. As an illustration, results for ${ }^{151} \mathrm{Sm}(\mathrm{n}, \gamma)$ reaction below $200 \mathrm{keV}$ are shown in Figure 2.

The calculated Maxwellian average capture cross section at $k T=30 \mathrm{keV}$ for ${ }^{151} \mathrm{Sm}(\mathrm{n}, \gamma)$ reaction is $3045 \mathrm{mb}$ (based on experimental data [8] and resonance parameters [3]), which is in excellent agreement with 3031(82) mb and 3100(160) mb measured by Wisshak et al. [9] and Abbondanno et al. [8], respectively. No uncertainties are available for ${ }^{151} \mathrm{Sm}$ evaluation in ENDF/B-VII library [4]. 


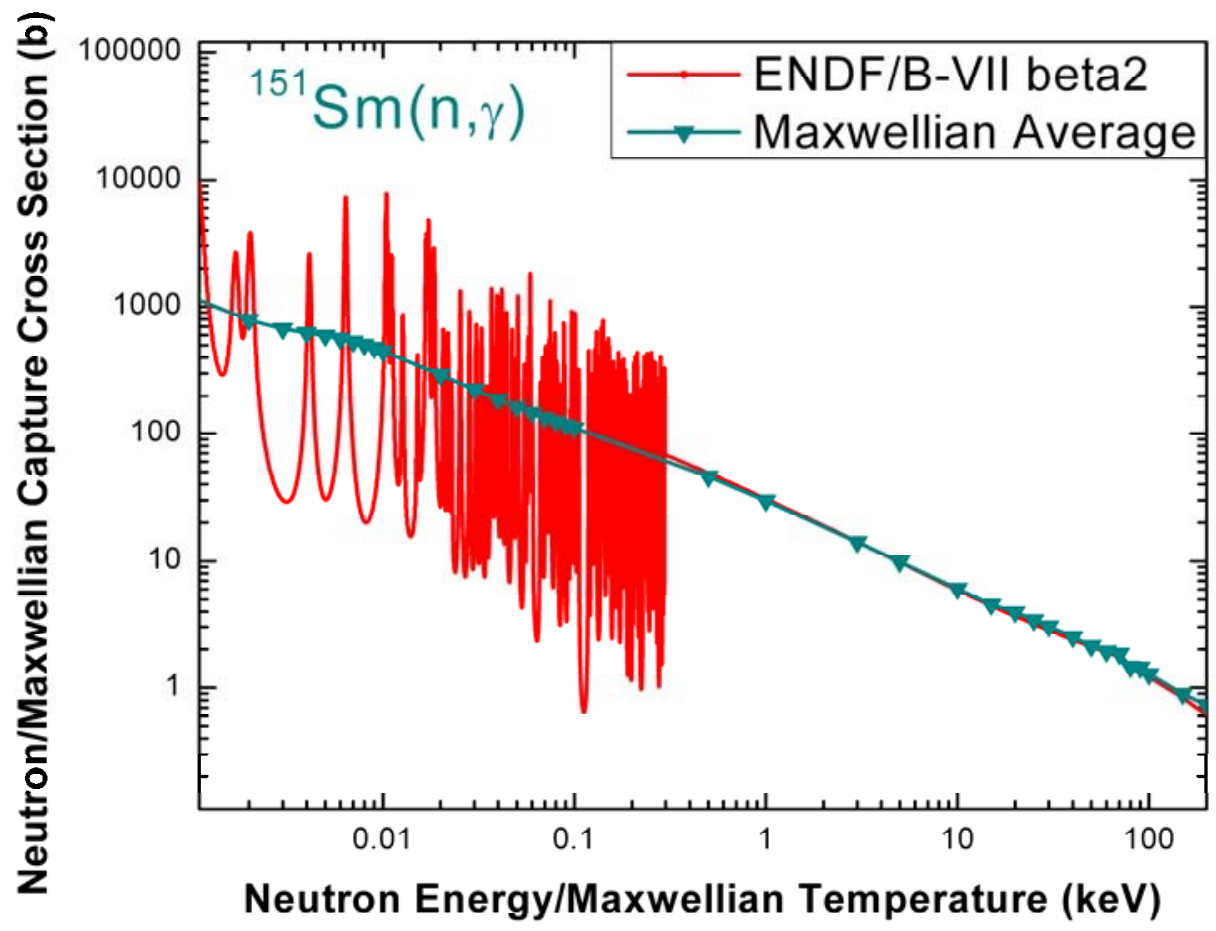

Figure 2. ENDF utility code INTER results for the ${ }^{151} \mathrm{Sm}(\mathrm{n}, \gamma)$ reaction. Maxwellian average neutron cross sections are shown in green while original ENDF/B-VII beta2 data are in red.

\subsection{Chart of Nuclides}

Chart of Nuclides ( $w w w . n n d c . b n l . g o v / c h a r t)$ is based on Evaluated Nuclear Structure Data File (ENSDF), and Nuclear Wallet Cards data and represents enormous resource for nuclear astrophysics. It provides information on almost all-known nuclei $(2,932)$, including some 140,102 levels, 204,017 $\gamma$-rays and 22,376 $\beta$ - and $\varepsilon / \beta+$ transitions. Related database NuDat 2.2 and highly popular Web service offer extensive options for data retrievals and nuclear level structure graphic capabilities.

\section{Publications}

Atlas of Neutron Resonances reference book [3] (www.nndc.bnl.gov/atlas) represents the neutron data for 98 elements and 486 isotopes (381 isotopes with resonance data). In addition to individual resonance parameters and thermal cross sections, it also includes evaluated $(\mathrm{n}, \gamma)$ cross sections for 186 isotopes at $30 \mathrm{keV}$. The cover of Atlas of Neutron Resonances is shown in Figure 3. 


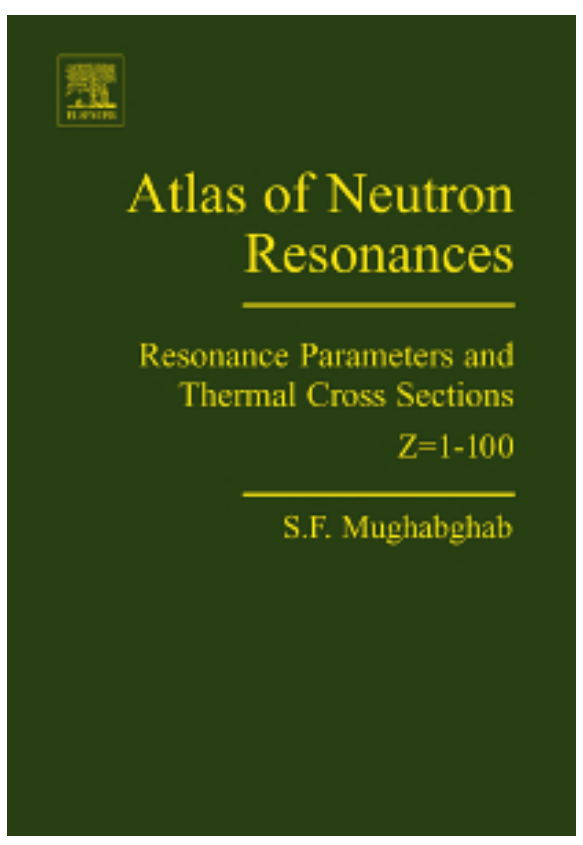

Figure 4. The Atlas of Neutron Resonances reference book [3].

\section{Conclusion}

The products of the NNDC and nuclear data services (www.nndc.bnl.gov) provide powerful resources for the astrophysics community. These data can serve as additional input for s-process nucleosynthesis calculations, particular in the energy region above $200 \mathrm{keV}$.

\section{Acknowledgements}

This work is supported by the Office of Nuclear Physics, Office of Science of the U.S. Department of Energy, under contract no. DE-AC02-98CH10886 with Brookhaven Science Associates, LLC.

\section{References}

[1] National Nuclear Data Center, Available from: http://www.nndc.bnl.gov

[2] B. Pritychenko, A.A. Sonzogni, D.F. Winchell, V.V. Zerkin, R. Arcilla, T.W. Burrows, C.L. Dunford, M.W. Herman, V. McLane, P. Oblozinsky, Y. Sanborn, J.K. Tuli , Nuclear Reaction and Structure Data Services of the National Nuclear Data Center, Annals of Nuclear Energy 33 (2006) 390.

[3] S.F. Mughabghab, Atlas of Neutron Resonances, Resonance Parameters and Thermal Cross Sections, $Z=1-100$, Elsevier 2006.

[4] M.B. Chadwick and CSEWG collaboration, Nuclear NDF/B-VII.0: Next Generation Evaluated Nuclear Data Library for Nuclear Science and Technology, to be published in Nuclear Data Sheets, December 2006. 
[5] B. Pritychenko, M.W. Herman, P. Oblozinsky, A.A Sonzogni, V. Zerkin, Nuclear Data Resources for Capture $\gamma$-Ray Spectroscopy and Related Topics, Proc. of $12^{\text {th }}$ Int. Conf. on Capture GammaRay Spectroscopy and Related Topics, Notre Dame, September 4-9, 2005, AIP Conference Proceedings 819 (2006) 128.

[6] C.E. Rolfs and W.S. Rodney, Cauldrons in the Cosmos, The University of Chicago Press, 1988.

[7] O. Ozer, Computer Code INTER, http://www.nndc.bnl.gov/nndcscr/endf/utility.

[8] U. Abbondanno and n_TOF Collaboration, Neutron Capture Cross Section measurement of ${ }^{151} \mathrm{Sm}$ at the CERN Neutron Time of Flight Facility (n_TOF), Phys. Rev. Lett. 93, 161103 (2004).

[9] K. Wisshak, F. Voss, F. Kaeppeler, M. Krticka, S. Raman, A. Mengoni, R. Galliano, Stellar Neutron Cross Section of the Unstable s-process Branching Point ${ }^{151}$ Sm, Phys. Rev. C 73, 015802 (2006). 